
$\left(\mathrm{XV}^{\mathrm{e}}-\mathrm{XIX}{ }^{\mathrm{e}}\right.$ siècle)

\title{
Les voyageurs britanniques et la pensée géographique sur les Alpes (1858-1899) : analyse linguistique en contexte de la topogénèse à la toponymation
}

\section{Samia Ounoughi}

\section{OpenEdition Journals}

Édition électronique

URL : https://journals.openedition.org/geohist/1036

DOI : 10.4000/geohist.1036

ISSN : 2264-2617

Éditeur

Association française de la Revue de géographie historique

\section{Référence électronique}

Samia Ounoughi, «Les voyageurs britanniques et la pensée géographique sur les Alpes (1858-1899): analyse linguistique en contexte de la topogénèse à la toponymation », Revue de géographie historique [En ligne], 17-18| 2020, mis en ligne le 07 juin 2021, consulté le 12 juin 2021. URL : http:// journals.openedition.org/geohist/1036 ; DOI : https://doi.org/10.4000/geohist.1036

Ce document a été généré automatiquement le 12 juin 2021

\section{(c) $(1)$}

Ce(tte) œuvre est mise à disposition selon les termes de la Licence Creative Commons Attribution Pas d'Utilisation Commerciale - Pas de Modification 4.0 International. 


\title{
Les voyageurs britanniques et la pensée géographique sur les Alpes (1858-1899) : analyse linguistique en contexte de la topogénèse à la toponymation
}

\author{
Samia Ounoughi
}

\section{Introduction}

1 Avec leurs nombreux récits de voyages dans les Alpes, les Britanniques ont largement contribué à la production de supports géographiques dédiés à la connaissance de la moyenne et de la haute montagne. Bien que le toit de la Grande-Bretagne s'élève à moins de 1400m d'altitude (le Ben Nevis en Écosse), les Britanniques figurent parmi les pionniers de l'alpinisme, une discipline à laquelle ils ont donné son nom et qui se développe notamment pendant la seconde moitié du XIX siècle. Leur pratique de la montagne se distingue aussi par le lien fondamental entre le pied et la plume, un lien d'ailleurs énoncé dans les règles mêmes de l'Alpine Club de Londres, le premier Club Alpin au monde, dès sa fondation en 1858 :

The interest shown by the Public in narratives of excursions through the less frequented districts of the Alps contained in several recent publications, had naturally suggested to others the idea of recording their adventures, either in separate volumes, or in the form of contributions to periodicals, when it was proposed that the facilities for combined action presented in the Alpine Club should be made use of to bring together, in a single volume, some of the materials likely to interest the general reader, which were available in the hands of several members of the Club. (Ball, 1859)

Aussi, les publications se multiplient-elles pendant la seconde moitié du XIXe siècle, suite à la création de la revue annuelle dans laquelle paraissent les récits d'ascension 
dans les Alpes. D'abord intitulée Peaks Passes and Glaciers, la revue qui paraît deux fois en 1859 et 1860 est publiée sous le titre The Alpine Journal à partir de 1863. C'est à la même époque que la première société savante britannique en géographie, fondée en 1830, devient la Royal Geographical Society (1859). À une époque où les grandes puissances économiques s'affairent à explorer le monde (Mary Kingsley, 1862-1900 en Afrique de l'ouest, David Livingstone 1862-1900 en Afrique...) et à le coloniser, les Britanniques montrent une conscience certaine de leur expérience du monde et de la nécessité de penser la géographie. Leur ambition est de mettre leur expérience en mots afin d'avoir la primeur de la production de la géographie, d'organiser le discours qui décrit l'espace et les territoires. Au-delà des récits qui paraissent dans la revue de l'Alpine Club de Londres, d'autres voyageurs publient des monographies dans lesquelles ils rapportent leurs randonnées et ascensions dans les Alpes, dans un mouvement d'anti-tourisme, qui loin des circuits balisés du Grand Tour, laisse à l'expérience de chacun le loisir de penser l'espace et d'en écrire la géographie. Zandski écrit d'ailleurs à propos de Alps and Sanctuaries de Samuel Butler (1835-1902) :

Alps and Sanctuaries is thus more than a signpost in the history of anti-tourism; it challenges the intellectual foundations of the structural Continental tour by encouraging the traveller to see for himself rather than 'by the book'. (Zdanski, 2007, 230).

L'objet de cet article est d'analyser le discours de ces récits afin d'en mettre au jour les traits saillants. La géo-graphie signifiant littéralement l'écriture de la Terre, les outils de la linguistique (linguistique de l'énonciation, sémantique) apporteront un éclairage sur la construction de la pensée géographique au sujet de la haute montagne alpine dans la seconde moitié du XIX ${ }^{e}$ siècle. Il s'agira de montrer comment se manifeste la pensée géographique des voyageurs britanniques dans les Alpes à travers leur métadiscours sur le voyage et la montagne. Après avoir montré le rôle central de l'écriture dans la démarche de réflexion sur les savoirs géographiques au sein de l'Alpine Club de Londres, nous analyserons deux caractéristiques notoires du discours produit dans la revue: le processus de création des toponymes que nous proposons d'appeler « toponymation » et le métadiscours sur la création des cartes géographiques.

\section{Ecrire l'espace - Penser la géographie}

4 Le discours de la géographie préoccupe les spécialistes de la discipline eux-mêmes depuis des décennies comme en témoignent Des mots et des lieux, la dynamique du discours géographique de Vincent Berdoulay (1988) ou plus récemment l'ouvrage collectif dirigé par Jean-François Staszak intitulé Le discours du géographe (1997). D’autres géographes, comme Pinchemel, mettent en avant l'histoire de la géographie comme une démarche d'écriture, et c'est en filant la métaphore de l'écriture qu'il définit l'histoire de la géographie.

Sur la surface de la Terre déjà rugueuse, déjà porteuse de signes de la Nature, les sociétés humaines, de millénaire en millénaire, ont écrit, réécrit, retouché, raturé, gommé. Elle en garde toujours les traces, les formes, pérennes mais aussi fossilisées; exhumées, transformées, ne subsistant parfois que par de simples toponymes. [...] La morphologie, dont les éléments nous parviennent depuis l'aube de l'humanité et s'édifient continuellement, livre la pensée des hommes, leur pensée géographique à qui sait la lire, la déchiffrer. La géographie est bien du temps matérialisé, de la pensée représentée, « interrestrée ». (Pinchemel, 441). 
5 Pinchemel rappelle ici le processus permanant par lequel les hommes modèlent et remodèlent la surface de la Terre en y laissant leurs traces, la transformant et la recréant dans une dynamique continue. En géographie alpine, c'est le même processus qui amène Debarbieux et Rudaz à nommer les habitants des montagnes et les voyageurs qui l'explorent «les faiseurs de montagne » (Debarbieux et Rudaz, 2010). A ces riches analyses sur le discours géographique il nous semble important d'ajouter une approche "d'observateur externe de cette discipline» (Sanguin, 1998), notamment celle de spécialistes du langage (Mondada et Racine, 1995). C'est ce que nous nous proposons ici d'aborder, du point de vue du linguiste, en prenant l'expression «faiseurs de montagne " au pied de la lettre, considérant que c'est l'expérience de la marche en montagne et l'écriture dont cette expérience est la trace qui façonnent la montagne, qui lui donnent son relief (Ounoughi, 2016).

6 C'est à ce double travail d'exploration et de construction du savoir sur la montagne que vont largement contribuer l'Alpine Club de Londres et sa revue annuelle. John Ball, premier président du Club est aussi le préfacier du premier numéro de la revue. Il indique la ligne éditoriale de production de savoirs scientifiques à partir de l'expérience de terrain, une ligne qui reste inchangée depuis la création du périodique. En ouverture de Peaks Passes and Glaciers il indique aux membres la voie à suivre pour produire du savoir géographique :

It was thought that many of those who have been engaged in similar undertakings, would willingly avail themselves of occasional opportunities for meeting together, for communicating information as to past excursions, and for planning new achievements; and a hope was entertained that such an association might indirectly advance the general progress of knowledge, by directing the attention of men, not professedly followers of science, to particular points in which their assistance may contribute to valuable results (Ball,1859, XII).

Usant de la voix passive (it was thought) pour indiquer que son énonciation porte la voix de tous les fondateurs du Club, John Ball élargit progressivement les fonctions pratiques d'entraide entre alpinistes qui justifient pour la revue une ambition mesurée mais réelle de contribuer par l'alliance de l'expérience et de l'écriture à la production d'un savoir géographique sur la montagne. Cette ambition (achievements; advance) tressée d'humilité (a hope was entertained; may contribute) est une conception originale du savoir géographique car le Club souhaite une production à la scientificité solide (advance the general progress of knowledge; valuable results) et pour ses fondateurs, la production de connaissances valables sur la montagne passe nécessairement par la contribution de tous, scientifiques ou non, la seule condition étant l'expérience de l'alpinisme et l'écriture de cette expérience (not professedly followers of science). C'est ce mélange d'érudition et d'expérience que le Club verse, par la revue, au patrimoine des savoirs géographiques.

8 À première vue, il semble ardu de donner une légitimité à la production d'un savoir construit à côté des disciplines telles que la géographie ou la géologie. L'équipe dirigeante de l'Alpine Club énonce pour ceci une ligne éditoriale qui guide le regard de l'alpiniste (by directing the attention of men, not professedly followers of science, to particular points in which their assistance may contribute to valuable results). Les membres du Club écrivent dans le sillage d'une sorte d'appel normé à contributions, travaillant ainsi à l'homogénéité de la revue par la longueur et le contenu de leurs récits.

9 L'humilité dans les propos de John Ball doit surtout être nuancée car les membres du Club ne sont pas de simples promeneurs. Ces alpinistes, dotés de qualifications 
universitaires pour la plupart, s'inscrivent aussi dans la longue tradition des sociétés savantes en Grande-Bretagne, qui remonte jusqu'à l'époque moderne avec la création en 1660 de la fameuse Royal Society dont J. Tyndall, A.C Ramsay font partie. À titre d'exemple, sur les 15 auteurs du premier numéro, 13 ont un titre universitaire (Bachelor of arts ou Master of arts) dont Wills, Mathews ou encore Ball et/ou sont membres d'au moins une société savante. John Ball est Membre de la Irish Royal Academy et de la Linnean Society. Ramsay est membre de la Geological Society, etc. Ces sociétés souhaitent produire des savoirs, les consigner, les conserver, et surtout les discuter. Au-delà des récits d'ascension, les nombreuses tables de mesure (barométriques, altitudinales, cianométriques, etc.) font de la revue un puits de savoirs scientifiques et géographiques sur la montagne. D'ailleurs, le titre complet de Peaks Passes and Glaciers, Excursions of Members of the Alpine Club devient The Alpine Journal, A Record of Mountain Adventure and Scientific Exploration by Members of the Alpine Club. Le Club asserte ici la volonté de promouvoir l'expertise et la réflexion de ses membres sur la géographie alpine et s'appuie sur l'Alpine Journal pour le diffuser. Cette approche fait la part belle à la géographie, une discipline qui s'affirme et s'institutionnalise à l'époque, mais aussi aux disciplines attenantes comme la géologie, l'hydrométrie, la glaciologie ou l'histoire naturelle.

10 La diversité des membres de ces sociétés savantes et de leurs spécialités au sein de l'Alpine Club va permettre une complémentarité des angles de vue. En outre, dès lors que le contenu de la revue est fondé sur l'expérience de ces alpinistes, il donne aussi davantage de liberté à chacun d'exprimer ce qu'il a perçu. C'est en étudiant la construction de ces expressions vives de la montagne que seront mis au jour quelques traits saillants de la manière dont la géographie prend forme dans les mots et dans quelle perspective le discours qui la sous-tend oriente, structure et conditionne la discipline elle-même.

\section{La toponymation : le tribut du vainqueur sommital ?}

11 Parmi les éléments linguistiques qui jalonnent la construction de la pensée géographique sur les Alpes, l'attribution des toponymes, plus précisément ici des oronymes, est une démarche fondamentale car elle relève de l'identification. Le corpus que nous avons construit à partir des articles de l'Alpine Journal constitue d'abord un riche creuset d'analyse de la topogenèse dès lors que l'on assiste au fil des mots à l'émergence d'oronymes nouveaux à mesure que les alpinistes atteignent de nouveaux sommets ou d'autres formes de reliefs. Alors que paraît Peaks Passes and Glaciers en 1858, nombreux sont les sommets et les cols qui n'ont pas encore été nommés. Entre 1858 et 1867, on rencontre au moins 15 occurrences de suggestions de nouvelles nominations de sommets ou de cols dans la revue du Club. S'il n'est pas question de toutes les citer, nous nous pencherons néanmoins sur quelques exemples prototypiques - c'est-à-dire ceux qui ont les caractéristiques les plus représentatives - où les alpinistes isolent, repèrent et sortent de leur environnement le référent de l'oronyme qu'ils proposent. C'est sur cette démarche liminaire de la naissance d'un oronyme que nous nous arrêterons, mais notons dès à présent qu'il subira ensuite l'épreuve de la reconnaissance sociale garante de son emploi pérenne et de sa fixation dans la nomenclature (Brunet et al., 1993, 485-486 ; Levy et Lussault, 2003, 926-927). C'est ce qui correspond en linguistique à la détermination maximale qui vient identifier et 
singulariser un référent extra-linguistique pour le cas qui nous concerne. La linguistique permet d'éclaircir la pensée sur le savoir géographique et sa production notamment par la toponymie. Dans le cadre de ces récits d'explorations des Alpes, il est nécessaire de parcourir le chemin qui mène au nouveau toponyme à la fois sur le terrain et dans le discours. C'est le parcours qui débute à la découverte d'un lieu et qui conduit à sa nomination, un parcours que je propose d'appeler «toponymation » et qui se situe en amont de la topogenèse.

12 La naissance d'un nom propre est le résultat d'une construction complexe ; il indique comment un savoir toponymique s'est élaboré. La méthode consiste à relever les traces de ces phénomènes de toponymation en analysant les marqueurs linguistiques qui instancient l'évolution du processus pour voir comment un lieu découvert reçoit un nom et comment ce dernier finit par être admis comme signifiant propre par la communauté linguistique. Ce cheminement linguistique avec ses détours par l'onomastique (surtout dans sa dimension diachronique) permet de mieux saisir les enjeux de la primauté de l'exploration avec comme finalité le modelage et la modélisation de la géographie. Par modelage nous renvoyons à l'expérience du pied qui foule le sol pour la première fois, à la main qui plante le piolet pour la première fois. Quant à la modélisation, elle est modalité linguistique, elle se forge dans l'écriture ; elle est le choix de la posture de l'énonciateur, le choix de ses mots et de leur agencement en discours pour dire la géographie et la penser tout à la fois (Ounoughi, 2016).

La toponymation est un processus performatif de nomination d'un lieu. A priori, le phénomène est simple. Une équipe est la première à choisir de donner un oronyme à une partie du relief et celui-ci qui serait ensuite porté sur les cartes. C'est le cas dans l'exemple suivant relaté par Alfred Wills. En 1857, Wills et sa cordée sont dans le massif $\mathrm{du}$ Mont Blanc et se dirigent vers le Glacier de Saleina en venant du Glacier du Tour. C'est cette étroite voie d'accès que Wills a proposé d'appeler Fenêtre de Saléna, aujourd'hui orthographiée Saleina ou Saleinaz. Wills precise que ce passage avait déjà été emprunté par Munier longtemps avant lui (sans préciser la date) et que Forbes avait fait ce trajet en 1850. Voici les propos de Wills sur la nomination de ce passage : "The gap itself was not more than four or five feet wide, so narrow and so definite, that, on W.'s calling it "la fenêtre," we adopted the name at once as being the most descriptive we could give it." (Wills, 1859, 17). Ici le choix du toponyme se fait par pure description, et de manière spontanée. L'un des membres de la cordée use du nom commun " fenêtre » et ce dernier, qui paraît une évidence géomorphologique et analogique pour le reste de la cordée, est adopté. « Fenêtre " passe ici du statut de nom commun à celui de nom propre dans le syntagme nominal "Fenêtre de Saléna" et rejoint les antonomases de type "aiguille» comme "Les Aiguilles Dorées». Ce nom propre a uniquement une fonction délocutoire, c'est-à-dire qu'il nomme en donnant une description. En effet, pour Mill (1843) comme pour Russell (1964), le nom propre constitue une description exacte laquelle n'est autre que le référent du nom propre luimême. Ainsi Mill pose l'individu (ici, le lieu géographique) comme le référent unique et entier du nom propre qui le désigne. Le sens du nom propre est donc son référent. Plus tard, Russell sera le premier à parler de description concernant les noms. Parmi eux, il assimile les noms propres aux adjectifs démonstratifs. Il définit le nom propre comme «Logically proper ", la description la plus parfaite du nom propre étant son référent. Notons au passage que, déplacé dans le milieu de la montagne, "fenêtre " attribue 
également une caractéristique de construction humaine à un milieu naturel, ce qui vient dénoter et connoter le modelage et la modélisation de la montagne par l'homme.

Si le phénomène de toponymation se produit de manière assez simple dans le cas de la Fenêtre de Saléna, il n'est ni une évidence linguistique, ni une évidence géographique. Françoise Armengaud suggère la possibilité que l'acte de nommer soit un échec. En effet, nommer, «transitivement, c'est l'acte de conférer un nom, de "baptiser" ». Cet acte, qui est de portée performative s'effectue selon des règles. Il n'est réussi ou acquis que si les conventions sont respectées : qui a autorité pour donner le nom ? Quel type de nom est conforme à la coutume? » (Armengaud, 2008-2009, 209). Si son propos porte sur le nom propre de personne, il vaut aussi ici pour le nom propre de lieu. C'est ce qui explique l'engagement prudent des alpinistes dans leur énonciation lorsqu'ils proposent des noms propres, faute de pouvoir tout simplement les imposer, comme John Ball en 1859 :

By analogy with the name of the nearest pass on the eastern side, and to commemorate the long range of dark rocks that leads towards it from the Breithorn, I proposed at the time that it should be called the Schwarz Thor, and have taken the liberty of preserving that designation in the title to this paper. (Ball, 1859, 128).

La mise en discours de la toponymation comme processus chez John Ball illustre bien la complexité de la démarche. Littéralement, on ne produit pas du savoir géographique par la seule légitimité de l'expérience ou par le privilège de la primauté de l'exploit. Ainsi, Ball ne dévoile pas directement le nom qu'il a retenu mais commence par expliciter la double logique géographique et diachronique de son choix qu'il inscrit dans un continuum et qui n'est donc pas une simple appropriation de la toponymation par goût personnel. Sur le plan modal, Ball prend ensuite une double précaution pour montrer qu'il n'entérine pas le nom qu'il a choisi. Il note "I proposed at the time that it should be called the Schwarz Thor". D'une part, la sémantique de "propose » rappelle que Ball ne se reconnaît pas la légitimité totale de la toponymation. Par l'emploi du prétérit simple il replace, d'autre part, cette suggestion à un moment antérieur à l'écriture. Cette variation dans les temps grammaticaux et l'usage du complément circonstanciel de temps (at that time) montrent que nous sommes en pleine liminalité toponymique. Autrement dit, la prise en compte de la diachronie indique que la toponymation est encore en cours d'élaboration. Entre la date de son excursion (du 17 au 19 août 1845) et le moment d'écriture (1846) qui diffère encore de la date de publication (1859), «Schwarz Thor » n'a pas encore acquis la stabilité du toponyme, c'est-à-dire, le nom propre d'un endroit précisément localisé, désigné, accepté et utilisé par toute la communauté linguistique (les habitants, les voyageurs, sur les cartes, etc.). Ce col situé à $3731 \mathrm{~m}$ d'altitude entre les sommets Pollux et Roccia Nera en Suisse valaisanne est resté et aujourd'hui orthographié Schwarztor. C'est parce qu'il sait que le processus de toponymation qu'il a lancé n'est pas parvenu à son terme que Ball s'investit pleinement dans son énonciation pour commenter son emploi du toponyme provisoire et il annonce qu'il a pris la liberté, comme il le dit, de conserver ce toponyme pour le titre de son article. Là encore, on assiste à une prise de précaution sémantique rappelant que Ball a conscience que ses mots ne sont pas encore stabilisés dans la communauté linguistique et culturelle, précaution sémantique assumée par l'emploi de l'aspect perfect «and have taken the liberty ». En effet, le perfect est un aspect qui valide un état de fait, à un moment donné, pour l'énonciateur l'« Aspect consistant, pour un énonciateur, à considérer comme pertinent(s), c'est-à-dire, significatif(s) à ses 
yeux à un moment d'énonciation donné un ou plusieurs événements révolu(s) ou partiellement révolu(s) à ce moment » (Groussier et Rivière, 1996). Le libre emploi d'un toponyme provisoire dans le titre de sa contribution est une décision antérieure à la publication de la revue, que l'auteur prend complètement à sa charge. Il justifie une démarche qui lui a semblée logique et pertinente au moment où il a intitulé son article, mais dont il se sait pour l'heure un usager isolé. La pertinence et la logique du choix s'instancient également dans son discours par l'emploi du present perfect dans une proposition à la fois indépendante et coordonnée à la précédente (and) indiquant un continuum plutôt qu'une assertion d'autorité isolée.

La démarche de suggestion du toponyme est également révélatrice. S'il s'était exprimé sur un mode purement indicatif, Ball aurait employé «I called it the Schwarz Thor ». Cependant, il passe d'abord par la voie passive : "that it should be called the Schwarz Thor". La voix passive signale que Ball ne se voit pas comme l'agent de la toponymation, que c'est aux autres (la communauté linguistique dont la tournure passive permet de faire l'élision) qu'appartient cette décision. Dans le même temps, il utilise la modalité déontique avec Should, utilisée "Lorsque la contrainte ou la possibilité sont de nature morale " (Lareya, 2004). Ball exprime une modalité de la personne, c'est-à-dire, un moyen par lequel il essaye d'influencer les personnes décisionnaires, sachant que lui n'a pas personnellement autorité à entériner un toponyme. Comment les convaincre? Par should (la forme passée de shall), Ball signale qu'il y a une nécessité de nommer l'endroit qu'il a découvert et que cette nécessité n'est pas l'expression de sa volonté, mais qu'elle lui est extérieure et supérieure. C'est le sens de shall, qui incarne ici toutes les données géographiques qui l'ont conduit à ce toponyme. Ainsi, cette forme grammaticale, bien qu'au passé, n'indique pas une antériorité, mais une relation contre-factuelle, donc non-réelle (Lapaire, R., Rotgé, 1991, 496) où le sujet et l'action de nommer le lieu Schwarz Thor n'est pas validée dans les faits à ce stade. Ceci lui permet d'employer le nom propre tout en signifiant que ce n'est pas (pour l'instant en tous cas) le nom du lieu qu'il désigne.

17 Ainsi, la toponymation, parce qu'elle est un processus de production de savoirs géographiques, est un acte de nomination particulièrement complexe. Elle ne saurait se résumer à une transmission du patronyme de l'alpiniste qu'elle dépasse largement, quand bien même on lui reconnait la primauté d'une ascension ou du passage d'un col. Il n'a pas pour tribut de son exploit la liberté totale de nommer ce sommet ou ce col. L'entrée d'un nom propre dans la langue implique la participation de toute la communauté linguistique: "La solidarité des registres allocutif et délocutif de la langue est fonctionnelle : la signification communiquée doit satisfaire simultanément à des conditions de succès, dont la sanction est la recevabilité par l'allocutaire de la forme de l'expression, et aussi des conditions de vérité. De ce point de vue, les interlocuteurs doivent se mettre d'accord aussi bien sur le sens à donner à leurs énonciations que sur leur référence possible. » (Jacques, 1979, 103).

Dans la plupart des cas, les alpinistes britanniques et irlandais ont proposé des toponymes dans les langues alpines: l'allemand, le français, l'italien. Ils ont donc respecté l'ancrage géographique, linguistique et culturel du lieu. L'identification d'un col ou d'un sommet de montagne à l'alpiniste peut survenir plus tard. Par exemple, Whymper qui accomplit le premier l'ascension du Cervin en 1865, relate son aventure dans Scrambles Amongst the Alps in the years 1860-69. Dans cet ouvrage, il ne fait jamais référence au couloir Whymper. La dénomination de sommets alpins par leur nom est 
une reconnaissance commune de l'appartenance culturelle totale de certains illustres alpinistes à ces lieux, une démarche qui salue leur contribution à la construction de la connaissance de la géographie alpine.

Octroyer un nom propre à un lieu est une démarche linguistique délicate qui s'inscrit dans un temps bien plus long que celui de l'énonciation. La production de savoirs et le discours qui en est la matière se fait dans un contexte géographique, culturel et linguistique que l'on ne peut ignorer, auquel cas le nom choisi resterait une occurrence valable que pour la seule personne qui l'a énoncée. Or, en dehors de toute interlocution, la nomination reste lettre morte et ne participe pas de la culture, ni du langage qui fonctionnent de pair. Ce phénomène, inscrit dans la langue, nous rappelle que si l'alpiniste parvient à gravir la montagne, celle-ci ne lui appartient jamais. Elle fait partie du patrimoine géographique des hommes.

\section{Le discours sur les cartes : une méta-réflexion sur la géographie}

Parmi tous les aspects de la réflexion sur la pensée dans la géographie alpine dans la revue du Club (métadiscours narratif, péritexte éditorial ou auctorial, discours sur la primauté de l'exploit...), le discours sur l'élaboration des cartes et son évolution est fondamental. En effet, certains articles de l'Alpine Journal (AJ) sont même exclusivement dédiés à la cartographie des Alpes (Pour exemple, R.C. Nichols, "Alpine Map of Switzerland", 1866). Partout ailleurs dans le corpus sous étude, la cartographie en cours d'élaboration est un sujet récurrent. Entre 1858 et 1870 on en retrouve au moins 20 occurrences de métadiscours sur les cartes en cours d'élaboration dans Peaks Passes and Glaciers (PPG) et l'AJ, et les exemples vont en nombres décroissants de 1870 à 1899, ce qui semble indiquer que les cartes des plus hauts massifs alpins se sont entre temps précisées et stabilisées. Sur le plan linguistique, il est d'autant plus important d'analyser le discours sur les cartes qu'il recroise ceux de la nomination ou toponymation, de la primauté de l'exploit et du métadiscours. Le discours sur la cartographie est aussi un métadiscours sur le savoir géographique. Il est d'autant plus présent dans les textes à un stade où l'on ne s'accorde pas encore sur les toponymes existants comme le montre cet exemple tiré d'un récit de Forster : "the Gross Ruchen, in the maps of Studer and Keller erroneously called Rüchi" (Forster, 1859, 373). On ne s'accorde pas non plus sur les toponymes à donner comment le voir avec dans la suite de son récit : "Professor Ulrich, who made the ascent from the Sand Alp [...] traversed some red snow, which, on the map he published of his route, he calls Schnee-Rosa." (ibid., 379). Les alpinistes ont donc besoin d'un double support de représentation: la carte et le commentaire de la carte qui exprime un savoir en construction et une toponymie en cours d'émergence ou de stabilisation.

21 Par ailleurs, le discours sur les cartes est empreint d'une multimodalité nécessaire de la transmission des savoirs à une époque où ils ne sont pas encore stables, les auteurs sollicitent donc des sources documentaires de natures diverses qui convergent pour identifier un même savoir. On constate aussi une forte dimension intertextuelle entre leurs récits car, en sus de l'aide apportée par les guides et les autres alpinistes, le discours des alpinistes-cartographes repose aussi sur l'aide qu'ils ont pu recevoir des militaires (Tuckett, 1864). On retrouve ces éléments dans le corps du texte sous forme de citations ou de références, ou dans des notes de fin d'articles. Ces notes sont souvent 
ajoutées par l'auteur ou par l'éditeur de la revue pour apporter un complément d'information acquis dans l'intervalle entre la soumission de l'article et sa publication. Les autres sources sont des articles de presse, des monographies publiées séparément, voire des témoignages oraux. Il existe notamment de nombreux commentaires portant sur la correction des cartes, comme c'est le cas de Charles Gilbert Heathcote en 1867.

Dans l'AJ, les auteurs ont la consigne d'être aussi concis que possible comme l'indique John Ball dans la préface de de PPG : "It was thought to be no slight advantage, that in this mode of publication the effort of each author would be rather to condense than to extend his narrative" (Ball, "Preface to the First Edition", 1859, XII). Dès lors, toute pause narrative dans l'histoire d'une expédition qui cède la place au commentaire en accroit la saillance (Genette, 1972). C'est le lieu de la réflexion sur les savoirs géographiques en cours de construction dont nous étudions ici un exemple chez Nichols :

I must add a few words respecting the map which illustrates this paper. In compiling it, I have derived most valuable assistance from information obtained by Mr. Reilly from the French Dépôt de la Guerre. The glaciers at the head of the Val de Tignes are from my own observations, and on the Italian side I have followed the Sardinian map, correcting it as far as I have been able. (Nichols, 1867, 118)

L'énonciateur s'investit davantage dans son discours pour prendre à sa charge à la fois la réflexion sur les savoirs qu'il a produits et leur caractère imperfectif. Nichols commente une carte de sa propre composition et engage son propos par l'emploi de la modalité radicale avec must dans: "I must add a few words respecting the map» (Sur l'évolution des sens de «Must ", voir Lowrey et Toupin, 2010). Cela permet de justifier qu'il commente par devoir, par obligation d'interrompre la narration pour expliquer un point qu'il est nécessaire de porter à la connaissance des lecteurs et qui respecte donc la double consigne éditoriale d'explicitation et de concision. Il reprend la genèse de sa carte qu'il désigne comme une compilation (in compiling it). La flexion -ing indique à la fois une action dans le cours de son déroulement et une visée, c'est-à-dire que Nichols se replace dans le contexte de l'élaboration de la carte, soulignant que, s'il s'agit d'un document publié, la carte conserve néanmoins son caractère si ce n'est inachevé, à tout le moins, perfectible. Ses choix linguistiques définissent sa carte comme la représentation d'un état du savoir géographique en construction dans un espace circonscrit et saisi à un moment précis. Il l'inscrit dans ce que nous pourrions désigner comme une pragmatique («LING., SÉMIOL. Qui étudie le langage du point de vue de la relation entre les signes et leurs usagers " (CNRTL).) de la cartographie qui rappelle le contexte géographique et diachronique de son travail et les autres acteurs qui ont participé à la construction de cet édifice déjà commencé avant lui. Cette généalogie inclue Franz Johann Joseph von Reilly (1766-1820), une sommité de la cartographie alpine à cette époque qui a produit plus de 830 cartes entre 1789 et 1806 pour son projet de Grand Atlas "Schauplatz der funf Theile der Welt", et remonte à la source primaire, le Dépôt de Guerre Français. Il ajoute les institutions locales avec la mappe sarde, vieille d'un siècle et demi et largement critiquée dans l'AJ. Conscient qu'un cartographe ne part pas seul dans son travail Nichols reprend donc son parcours cartographique avant d'expliquer sa contribution à la cartographie, gagnant ainsi de la légitimité.

L'emploi du present perfect à trois reprises (I have derived; I have followed; as far as I have been able) fait résonner/raisonner en miroir la production du discours géographique et la production des cartes. Nichols insiste sur le résultat de sa recherche, au moment où il produit son article. Il signale ainsi que la carte est la trace d'un réseau de 
connaissances, pas toujours fiables, et qu'elle n'est qu'un bilan intermédiaire à repenser, à retravailler. Même les corrections qu'il a pu apporter à la mappe sarde sont énoncées avec beaucoup de prudence (as far as I have been able). Au final, dans la production de cette carte, il y a plus de références à des apports antérieurs qu'à ceux de l'auteur, qui se résument à deux éléments: la correction de la mappe sarde et son observation du haut du glacier du Val de Tignes. Ce dernier est aussi son seul emploi du présent simple à la forme purement indicative (The glaciers at the head of the Val de Tignes are from my own observations). L'assertion simple et directe est donc utilisée de manière parcimonieuse et souligne surtout la responsabilité qu'il prend dans sa participation à l'élaboration cartographique, rendant son apport personnel provisoire car soumis à l'appréciation des lecteurs, au premier rang desquels les membres du Club.

Tous ces ressors linguistiques convergent pour apporter des types d'informations complémentaires et nous indiquer de quelle manière l'auteur de l'article de l'AJ, cartographe et alpiniste, membre de l'Alpine Club, se situe face à la composition d'une nouvelle carte de géographie. Ils témoignent des acquis et les besoins en matière de production de cartes pour les alpinistes et surtout de la grande prudence nécessaire face à la fragilité des savoirs en construction. L'enjeu est de taille car cette carte va servir aux futurs alpinistes et aura donc potentiellement un impact sur la faisabilité et la réussite de leur(s) expédition(s) selon la justesse des informations qui y auront été apportées.

\section{Conclusion}

Les excursions des membres de l'Alpine Club, si elles sont des chapitres séparés, n'en demeurent pas moins inscrites dans un continuum de recherches et de construction collective des connaissances en géographie alpine doublées d'une méta-analyse des savoirs en constante évolution. Le choix des éléments linguistiques qui mettent les savoirs géographiques en mots est en soi-même une pensée sur le savoir géographique. Un col traversé avec succès, une ascension qui relève de l'exploit n'ouvre aucun droit à l'alpiniste d'imposer un nom à la montagne arbitrairement et solitairement. Il est là pour inscrire un savoir nouveau et le processus de toponymation dans sa complexité vient rappeler à quel point cette démarche ne saurait se défaire de l'empreinte de l'histoire culturelle et géographique locale. La narratologie nous montre, quant à elle, sur quel mode l'alpiniste choisit de parler. La modalité sous toutes ses formes permet de dire le degré d'implication de l'énonciateur dans son discours depuis le point de vue le plus indicatif jusqu'au plus commentatif. Les aspects perfect et -ing montrent l'état de l'art à un moment précis de l'histoire et à quel point l'alpiniste-auteur est conscient du contexte contraignant dans lequel il produit des savoirs. Il note aussi combien il est conscient de la valeur perfectible de ceux-ci, parce que les méthodes de recherche et les outils évoluent. C'est aussi parce que la montagne est un milieu à aux temporalités multiples Il est un temps géologique long qui dépasse l'humain et lui permet l'exploration d'un milieu stable. Il est aussi une temporalité du climat qui varie d'heure en heure où les impasses peuvent devenir voies et inversement (Ounoughi, 2013). Le ing de la visée incarne ici l'ambition de préciser les savoirs, la prudence de la démarche dans leur production et l'humilité face à une visée qui reste le seul statut pérenne possible car en dernière instance il n'est pas de connaissance parfaite de la montagne. L'aventure de ceux qui la pratiquent est une pierre apportée à l'édifice des savoirs et 
elle reste une expérience unique qui enjoint à penser constamment savoirs géographiques. C'est cette fonction que le langage recouvre et c'est son analyse qui la met au jour.

\section{BIBLIOGRAPHIE}

Alpine Journal (The), vol. I-XIX, vol. I 1863-1864, vol. II 1865-66, vol. III 1867, vol. IV 1868-1870, vol. V 1870-1872, vol. VI 1872-1874, vol. VII 1874-1876, vol. VIII 1876-1878, vol. IX 1878-1880, vol. X $1880-1882$, vol. XI 1882-1884, vol. XII 1884-1886, vol. XIII 1886-1888, vol. XIV 1888-1890, vol. XV 1890-1891, vol. XVI 1892-1893, vol. XVII 1894-1895, vol. XVIII 1896-1897, vol. XIX 1898-1899.

Ball J., 1859, « Preface to the First Edition », Peaks, Passes and Glaciers, London, Longman, Green, Longman, and Roberts, p. V-XII.

Ball J., 1859, « Pass of the Schwartz Thor. Zermatt to Ayas », Peaks, Passes and Glaciers, London, Longman, Green, Longman, and Roberts, p. 155-193.

Berdoulay V., 1988, Des mots et des lieux, la dynamique du discours géographique, Paris, C.N.R.S. Éditions.

Bourdon É., 2011, Le voyage et la découverte des Alpes. Histoire de la construction d'un savoir (1492-1713), Paris, Presses Universitaires de la Sorbonne.

Brunet R., Ferras, R. et Théry, H., 1993, Les mots de la géographie. Dictionnaire critique, Montpellier, RECLUS ; Paris, la Documentation française.

Butler S., 1986 [1881], Alps and Sanctuaries of Piedmont and the Canton Ticino, London, Allan Sutton.

Debarbieux B., Rudaz G., 2010, Les faiseurs de montagne. Imaginaires politiques et territorialités : XVIII ${ }^{e}$ $\mathrm{XXI}{ }^{e}$ siècle, Paris, CNRS.

Forster R. W. E., 1859, «The Baths of Stachelberg, and the Heights and Passes in the Vicinity ", Peaks Passes and Glaciers, London, Longman, Longman, and Roberts, p. 371-399.

Genette, G., 1972, Figures III, Paris, Seuil.

Grell C. Halleux, R., 2016, Sciences, Techniques, pouvoirs et sociétés du XV siècle au XVIII siècle. Paris, Armand Collin.

Groussier M.-L., Rivière, C., 1996, Les Mots de la linguistique : lexique de linguistique énonciative. Paris, Ophrys.

Guillemette L., Lévesque C., 2016, « La narratologie », Louis Hébert (dir.), Signo [en ligne], Rimouski (Québec), http://www.signosemio.com/genette/narratologie.asp. (site consulté le 12 mai 2018).

Heathcote C. G., 1867, « The Col de Pierre Joseph », The Alpine Journal, vol. III, 1867, London, Longman, Reader, and Dyer, p. 99-104.

Jacques F., 1979, Dialogiques : recherches logiques sur le dialogue, Paris, P.U.F.

Lapaire R., Rotgé, W., 1991, Linguistique et grammaire de l'anglais, Toulouse, P.U.M. 
Lareya P., 2004, «L'expression de la modalité en français et en anglais (domaine verbal) », Revue belge de philologie et d'histoire, Langues et littératures modernes - Moderne taal en litterkunde, $\mathrm{p}$. 82-3, 733-762.

Mondada L., Racine J.-B., 1995, « Géographie et sémiolinguistique », A. Bailly, R. Ferras et D. Pumain (dir.), Encyclopédie de géographie, Paris, Economica, p. 239-254.

Lowrey B., Toupin, F., 2010, "L'invariant à l'épreuve de la diachronie », Corela [En ligne], 8-2, mis en ligne le 26 novembre 2010, consulté le 13 mai 2018. URL : http://journals.openedition.org/ corela/1853; DOI : 10.4000/corela.1853

Mill J.-S., 1843, A System of Logics, vol. 1. London, John W. Parker, West Strand.

Nichols R. C., 1866, « Alpine Map of Switzerland », The Alpine Journal, vol. II, 1865-66, London, Longman, Reader, and Dyer, p. 350-352.

Nichols R. C., 1867, « Excursions in the Graians ( $\left.{ }^{\circ} 3\right)$. Ascent of the Albaron », The Alpine Journal, vol. III, London, Longman, Reader, and Dyer, p. 105-118.

Ounoughi S., 2013, « The Swiss Alps and Character framing in No Thoroughfare by Wilkie Collins and Charles Dickens, 1894 », Leroy, M. (dir.), Dickens and Europe. Cambrigde Scholars Publishing, New Castle upon Tyne, p. 114-125.

Ounoughi S., 2016, "La saillance et le discours sur le relief », Journal of Alpine Research / Revue de géographie alpine [En ligne], 104-2, mis en ligne le 21 septembre 2016, consulté le 29 avril 2018. URL : http://journals.openedition.org/rga/3381 ; DOI : 10.4000/rga.3381

Ounoughi S., 2017, « Analyse du discours de la liminalité : Butler, de l'autre côté du Saint-Gothard entre passage et ancrage ", ILCEA [En ligne], 28, mis en ligne le 06 mars 2017, consulté le 28 avril 2018. URL : http://journals.openedition.org/ilcea/4099

Pinchemel P. et G., 1992, La Face de la Terre : éléments de géographie, Paris, Armand Collin.

Russell B., 1964, [1956], « On Denoting », Logic and Knowledge Essays 1901-1950 », London, George Allen \& Unwin ltd, p. 39-57.

Sanguin A.-L., 1998, « La discipline géographique et ses discours », Annales de géographie, p. 335-336 (CR de Jean-François Staszak, Les discours du géographe, 1997).

Saussure F., 1968, Cours de linguistique générale, Wiesbaden, Harrassowitz, 2.

Sprat T., 1667, The History of the Royal Society of London, For the Improving of Knowledge. London, T. R. for F. Martyn at the Bell.

Staszak J.-F. (dir.), 1997, Le discours du géographe, Paris, l'Harmattan.

Tuckett F. F., 1864, « Explorations in Alps of the Dauphiné », The Alpine Journal, vol. I, 1863-64, London, Longman, Longman, and Roberts, p. 145-183.

Viviès J., 2016, Revenir / devenir. Gulliver ou L'Autre Voyage. Paris, Éditions rue d'Ulm, Presses de l'École Normale Supérieure, « Offshore ».

Weld C. R., 1848, A History of the Royal society with Memoirs of the Presidents. Compiled from Authentic Documents, London, John W. Parker, West Strand, vol. I.

Whymper Ed., 1871, Scrambles Amongst the Alps in the years 1860-69, London, J. Murray.

Wills A., 1859, « Passage of the Fenêtre de Saléna, from the Col de Balme to the Val Ferret, by the Glacier du Tour, the Glacier de Trient, and the Glacier de Saléna ", Peaks Passes and Glaciers, London, Longman, Green, Longman, and Roberts, p. 1-38. 
Zdanski C., 2007, « Samuel Butler, Local Identity, and the Periodizing of Northern Italian Art: The Travel Writer-Painter's View of Art History », Paradis, J. (dir.), Samuel Butler, Victorian against the Grain: a Critical Overview, Toronto, University of Toronto Press, p. 223-250.

\section{RÉSUMÉS}

Le premier Club Alpin au monde, The Alpine Club of London, est fondé en 1857 et avec lui, la revue du Club d'abord intitulée Peaks Passes and Glaciers. Elle est publiée pour la première fois en 1858 et deviendra The Alpine Journal à partir de 1863. Composée en grande partie de récits de voyages et d'explorations à visée scientifique, la revue est un patrimoine précieux qui nous informe sur la construction des savoirs géographiques sur les montagnes et sur la réflexion qui accompagne l'émergence de ces savoirs. Le corpus étudié se compose des numéros de la revue du Club Alpin de Londres depuis sa première parution (1858) jusqu'à la fin du XIX ${ }^{\mathrm{e}}$ siècle (1899). Les productions toujours plus nombreuses des auteurs et membres du Club sont un marqueur fort de l'accélération de l'exploration des hautes montagnes, notamment dans l'arc alpin, espace auquel cet article est consacré. Ces textes présentent le double intérêt de répondre à une ligne éditoriale stricte de concision et de précision scientifique tout en laissant à chaque auteur une liberté de style pour exprimer son vécu sur le terrain. Le but de cet article est donc de mener une analyse du discours de ces alpinistes pour sonder, à travers l'observation de leurs choix linguistiques et de l'agencement discursif de leur récit de quelle manière ils mènent une réflexion sur les savoirs géographiques en construction, du piolet à la plume. Les sujets de ces textes convoquent naturellement une approche interdisciplinaire. L'histoire et la géographie viennent ici contextualiser et éclairer les analyses du discours en anglistique. Les outils de la pragmatique (linguistique de l'énonciation, sémantique, narratologie) apporteront un éclairage sur la construction de la pensée géographique au sujet de la haute montagne alpine dans la seconde moitié du XIX ${ }^{e}$ siècle. Après avoir montré le rôle central de l'écriture dans la démarche de réflexion sur les savoirs géographiques au sein de l'Alpine Club de Londres dans la première partie, nous analyserons deux caractéristiques notoires du discours produit dans la revue: le processus de création des toponymes dans la seconde partie et le métadiscours sur la création des cartes géographiques dans la troisième. Si La géo-graphie est littéralement l'écriture de la terre, il est nécessaire de sonder la construction de ce discours pour mieux comprendre l'agencement de la pensée géographique dont le processus complexe appelle à la fois la prudence de l'alpiniste, la rigueur du scientifique et la créativité de l'écrivain.

The first Alpine Club in the world was founded in London in 1857. The same year, the founding members created a yearly periodical to record the alpinists' experiences of their expeditions. It was entitled Peaks, Passes and Glaciers and was first published in 1858. In 1863 its title was changed to The Alpine Journal. As the issues of this periodical are mainly composed of travel and exploration narratives with a scientific perspective, the whole corpus is a precious heritage, a rich source of information on the shaping of geographic knowledge about the mountains and the reflection on the emergence of this knowledge. The corpus under study is a collection of the issues of the periodical since its creation to the end of the $19^{\text {th }}$ century (1858-1899). The ever growing number of texts produced by the members of the Alpine Club highlights the expansion of mountain exploration at high altitudes, in particular in the Alpine Arch, the mountain chain which this article is dedicated to. These narratives present a two-fold interest in so far as the authors abide by strict editorial rules on the one hand, while still writing freely in their own style to express their unique experience on the ground, on the other.The aim in this article is to carry out a linguistic analysis of these alpinists' discourse. We will observe their linguistic choices as well as the narrative construction of their texts to search the traces of their reflection on 
geographic knowledge. How is the emergence of new geographic knowledge shaped in the alpinists' discourse while this knowledge is yet still fleeting from step-cutting to text-writing? The various topics of these narratives naturally require an interdisciplinary approach. History and geography are seminal to contextualise discourse analysis and shine light on it. The tools of pragmatics (enunciation linguistics, semantics, narratology) will shed light on the shaping of geographic thought about Alpine regions at high altitudes in the second half of the 19th century. I will first show the crucial part played by writing in the reflexive process of geographic knowledge within the Alpine Club of London. The second part will then consist in the analysis of salient linguistic characteristics of the discourse produced in the periodical: the process of placenaming. The third part will focus on the metadiscourse about mapping in process. While geography literally refers to the act of writing about the earth, it is necessary to inquire on the linguistic construction to better understand the shaping of geographic reflection, the process of which is complex. Indeed, it requires the carefulness of the alpinist, the scientific minuteness of the researcher and the creativity of the writer.

\section{INDEX}

Mots-clés : Alpes, Alpine Journal, analyse du discours, linguistique, récits de voyage, toponymation, savoirs géographiques, XIXe siècle

Keywords : Alps, Alpine Journal, discourse analysis, geographic knowledge, linguistics, toponymation, travel narratives, 19 th century

\section{AUTEUR}

\section{SAMIA OUNOUGHI}

Maître de Conférences section 11, études anglophones, LIDILEM EA609, 46 rue du général Ferrié

38100 Grenoble

Laboratoire Lidilem

Université Grenoble Alpes

Bâtiment Stendhal

CS40700

38058 Grenoble cedex 9

samia.ounoughi@univ-grenoble-alpes.fr 